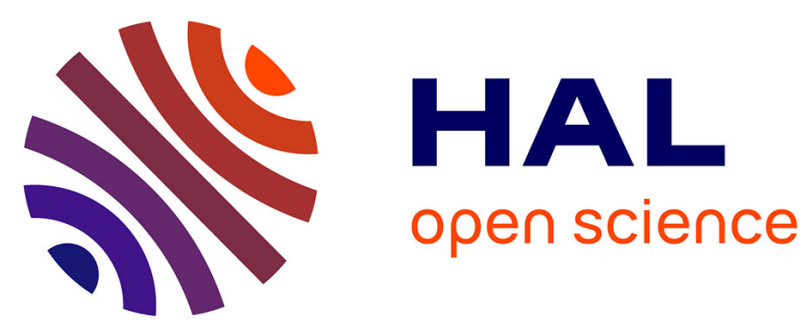

\title{
Density and flow reconstruction in urban traffic networks using heterogeneous data sources
}

Andres Ladino Lopez, Carlos Canudas de Wit, Alain Kibangou, Hassen Fourati, Martin Rodriguez-Vega

\section{- To cite this version:}

Andres Ladino Lopez, Carlos Canudas de Wit, Alain Kibangou, Hassen Fourati, Martin RodriguezVega. Density and flow reconstruction in urban traffic networks using heterogeneous data sources. ECC 2018 - 16th European Control Conference, Jun 2018, Limassol, Cyprus. pp.1-6. hal-01731356

\section{HAL Id: hal-01731356 https://hal.science/hal-01731356}

Submitted on 14 Mar 2018

HAL is a multi-disciplinary open access archive for the deposit and dissemination of scientific research documents, whether they are published or not. The documents may come from teaching and research institutions in France or abroad, or from public or private research centers.
L'archive ouverte pluridisciplinaire HAL, est destinée au dépôt et à la diffusion de documents scientifiques de niveau recherche, publiés ou non, émanant des établissements d'enseignement et de recherche français ou étrangers, des laboratoires publics ou privés. 


\title{
Density and flow reconstruction in urban traffic networks using heterogeneous data sources
}

\author{
Andres Ladino, Carlos Canudas-de-Wit, Alain Kibangou, Hassen Fourati, and Martin Rodriguez *
}

\begin{abstract}
In this paper, we consider the problem of joint reconstruction of flow and density in a urban traffic network using heterogeneous sources of information. The traffic network is modeled within the framework of macroscopic traffic models, where we adopt Lighthill-Whitham-Richards model (LWR) conservation equation characterized by a piecewise linear fundamental diagram. The estimation problem considers two key principles. First, the error minimization between the measured and reconstructed flows and densities, and second the equilibrium state of the network which establishes flow propagation within the network. Both principles are integrated together with the traffic model constraints established by the supply/demand paradigm. Finally the problem is casted as a constrained quadratic optimization with equality constraints in order to shrink the feasible region of estimated variables. Some simulation scenarios based on synthetic data for a manhattan grid network are provided in order to validate the performance of the proposed algorithm.
\end{abstract}

\section{INTRODUCTION}

Accurate state information of the network provides an empowering tool for decisions about the usage of the traffic infrastructure. Efficient road traffic management policies strongly depend on the vehicle density information. Road vehicle density (or road occupancy), i.e. number of vehicles per kilometer, is a critical parameter regarding various aspects: in road maintenance and traffic monitoring, it is essential to inform the state of the network and to perform preventive maintenance; in designing traffic light control policies, its evolution is essential to construct efficient feedback laws, see [10]. Despite the development of a large variety of sensing technologies, measuring vehicle density is still a difficult task in complex traffic networks. Magnetic loop detector (MLD) has been the traditional technology but it is very expensive for both deployment and maintenance. The emergence of new technologies such as Floating car data (FCD) has reduced the cost of measurement while increasing the amount of traffic monitored. However, FCD measures average velocity in a road sector (not densities), often suffers from a limited penetration rate, and does not suffices alone for a correct reconstruction of vehicle density. The problem of traffic state reconstruction has been widely investigated in the literature. Works in [19], [20], [21], [2], [15] are some examples where Luenberger observers and Kalman filter (KF) like techniques are applied to reconstruct densities and speeds. Data fusion techniques are explored in [7] where multiple sources of data are integrated in the three

\footnotetext{
* The authors are with Univ. Grenoble Alpes, CNRS, Inria, Grenoble INP, GIPSA-Lab, 38000 Grenoble, France \{andres.ladino-lopez, carlos.canudas-de-wit, alain.kibangou,

hassen.fourati, martin.rodriguez-vega\}@gipsa-lab.fr
}

detector model. In more recent works [13], [14], authors proposed to combine both FCD and MLD for flow and density estimation. The study in [1] considers sources of connected vehicles and not connected ones for the density reconstruction. A different approach [4] formulated the problem for lagrangian and loop data integration into a model from variational calculus perspective. For a more general reviews on this problem, see [8], [18]. This paper is devoted to the problem of joint reconstruction of flow and density in traffic networks using heterogeneous data sources. To the best of our knowledge, this problem has been partially treated in some simple scenarios like highways, but very seldom in urban traffic networks. The estimation process is carried out on the basis of the steady-state equilibrium of traffic network with an explicit model for the road intersections. Each single road section is modeled by the well known macroscopic fluid traffic model LWR, while the intersections are modeled by solving the single junction problem [3] as a set of optimization problems (maximum outflows) which turn out to be Linear Programming (LP) if the piece-wise linear fundamental (triangular) diagram is adopted.

The whole estimation problem is finally described as a minimization of the quadratic error between the measurement and their estimates under linear equality constraints coming from the network model. One key general difficulty in this problem, which is inherent to the nature of the system, is the "non-invertibility" of the velocity-to-density map in free-flow conditions. Namely, several density values are possible when the network operate in free-flow velocity. Then, in this freeflow regime, one velocity point maps to a bounded density set. Although several regularization schemes are possible, the results still remain very sensitive to noise in this domain, and the errors can be spread out to the whole set of estimated variables. To overcome this difficulty, we make use of the equilibrium state of the system which intends to provide flow propagation within the network and we adopt strict equality constraints which allows the density recovery by promoting solutions in the fundamental diagram.

The paper is organized as follows. We first introduce the network traffic model adopted in this paper and formulation the problem under study in Sections II and III respectively. In Section IV, we presents the optimization algorithms for both the density and flow reconstructions which are evaluated in Section $\mathrm{V}$ for a given scenario.

\section{ROAD TRAFFIC NETWORK MODEL}

A urban network is made of roads and junctions between them. In what follows, we describe the dynamics governing 
each road and their splitting or merging in junctions.

\section{A. Macroscopic Traffic model for a single section}

The most used instance of a continuous macroscopic traffic model is the Lighthill-Whitham-Richards model (LWR) [12], [17], which describes the spatio-temporal evolution of vehicle density $\rho(x, t)$ and flow $\Phi(\rho(x, t))$ as:

$$
\frac{\partial}{\partial t} \rho(x, t)+\frac{\partial}{\partial x} \Phi(\rho(x, t))=0,
$$

The characteristic curve of $\Phi(\rho)$, widely known as fundamental diagram, may take multiple forms including a triangular one [16]. The discretized version of this model is known as the Cell Transmission Model (CTM) [5], [6], which is undoubtedly one of the main and most well known traffic models to date. It is based on a first order Godunov approximation of LWR [11].

In CTM, the road segment of interest is first partitioned into a sequence of cells. The propagation of traffic dynamics in each cell is given by the following set of equations:

$$
\begin{aligned}
\rho_{i}(k+1) & =\rho_{i}(k)+\frac{T}{l_{i}}\left(\varphi_{i-1}(k)-\varphi_{i}(k)\right) \\
\varphi_{i-1}(k) & =\min \left(v^{\text {free }} \rho_{i-1}(k), \varphi^{\max }, w\left(\rho^{\max }-\rho_{i}(k)\right)\right)
\end{aligned}
$$

where $\rho_{i}(k)$ denotes the current vehicles density in the $i$ th cell while $\varphi_{i}(k)$ stands for the interface flow between the $i$ th and $(i+1)$ th cells, $l_{i}$ being the length of the cell. The followings are the set of parameters associated to each cell ${ }^{1}$ :

- $\rho^{\max }$, the maximum density, often referred as jam density,

- $\varphi^{\max }$, the maximum capacity flow,

- $v^{\text {free }}$, the maximum velocity of vehicles in the cell, said the free-flow velocity,

- $w$, the speed of the congestion wave in back propagation.

These parameters can be easily found in the fundamental diagram (See Fig. 1).

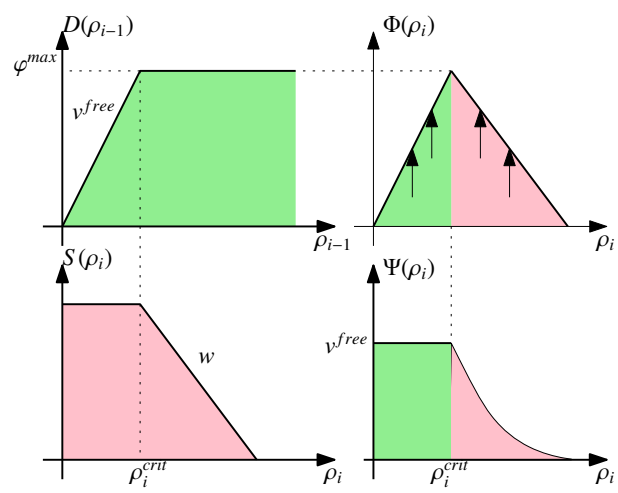

Fig. 1: Left - Demand and Supply functions. Right - Densityflow and density-speed fundamental diagrams where $\rho^{\text {crit }}$ stands for the critical density which represents the switching point between free-flow and congested regimes.

\footnotetext{
${ }^{1}$ We omit the $i$ index in the notation of parameters of each cell to simplify the reading process.
}

\section{B. Junction model}

Let $\mathcal{G}$ denote the set of junctions within the network. A junction labeled $g \in \mathcal{G}$ represents a physical connection between 2 or more roads and they can be found in shapes of bottlenecks, divergences, or merges. A junction $g$ is represented by the tuple $\left(\mathcal{I}_{g}, \mathcal{R}_{g}\right)$ where $\mathcal{I}_{g}=\left\{I_{i}: i=1, \ldots, n+m\right\}$ represents a set of roads and $r_{[i j]} \in \mathcal{R}_{g}$ are the splitting ratios denoting driving preferences. Each element of $\mathcal{G}$ symbolizes the existing junction between the set of $n$ upstream roads $\mathcal{I}_{g}^{-}=\left\{I_{i}: i=1, \ldots, n\right\}$ and the set of $m$ downstream roads $\mathcal{I}_{g}^{+}=\left\{I_{j}: j=n+1, \ldots, n+m\right\}$ as depicted in Fig. 2. The set of junctions $\mathcal{G}$ along with the corresponding sets $\mathcal{I}_{g}^{-}, \mathcal{I}_{g}^{+}, \mathcal{R}_{g}$ constitute the called Road Traffic Network (RTN).
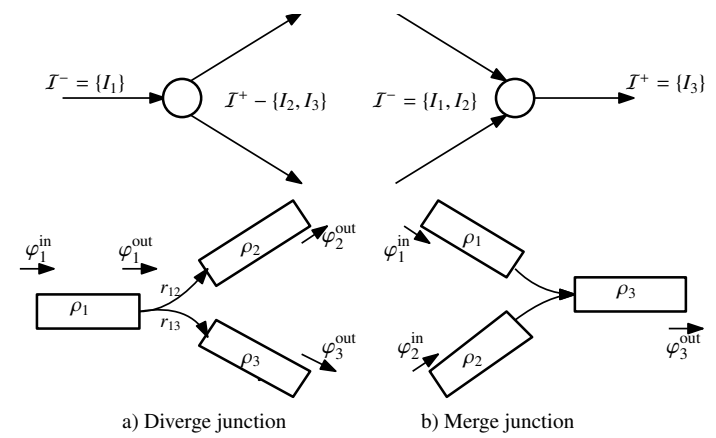

Fig. 2: Simplified case of junction types

Each cell represents a node in a network and it is characterized by a set of unique variables describing the macroscopic behavior: $\rho_{i}(k)$, the density, $\varphi_{i}^{\text {in }}(k)$, the ingoing flow, and $\varphi_{i}^{\text {out }}(k)$, the outgoing one. We refer to $\rho(k), \varphi^{\text {in }}(k), \varphi^{\text {out }}(k)$ as the vectors collecting all the densities and flows of the network.

Definition 1 (Single Junction Problem): Consider a junction $g$ with $n$ incoming roads $\mathcal{I}_{g}^{-}$and $m$ outgoing ones $\mathcal{I}_{g}^{+}$ under the vehicle conservation law, i.e.

$$
\sum_{i=1}^{n} \varphi_{i}^{\text {out }}\left(\rho_{i}(k)\right)=\sum_{j=n+1}^{n+m} \varphi_{j}^{\text {in }}\left(\rho_{j}(k)\right) .
$$

The single junction problem consists in determining values of inflow and outflow consistent with the solutions of the LWR model (or its discretized counterpart (2)).

Since solutions of $\varphi^{\text {in }}, \varphi^{\text {out }}$ to the traffic distribution problem in (3) are non unique, additional rules to incorporate drivers' behavior can be added to define a particular solution. Typical rules for Manhattan-like networks ${ }^{2}$ are:

A) Drivers follow fixed routes. Hence there exists traffic routing coefficients $r_{i j} \in(0,1]$, representing the splitting ratio from road $I_{i}$ to $\operatorname{road} I_{j}$. The resulting matrix, called Splitting Ratio matrix, $R_{g}=[r]_{i j}$ is row stochastic $\sum_{j=n+1}^{n+m} r_{i j}=1$. This matrix is assumed to be known (at least in average).

${ }^{2}$ For the sake of simplicity we have limited our approach to Manhattanlike networks where there are no merging junctions in which $n>m$. However, it is also possible to generalize for any other type of networks including also merging junctions by adding priority driving rules, see [9],[3] 
B) Drivers tend to maximize the network throughput. So drivers behavior is such that incoming flows to the junctions $\varphi^{\text {out }}$ are maximized.

A solver for the junction problem: Consider the rules A) and $\mathrm{B}$ ) together with the triangular fundamental diagram of Fig. 1 having the density-flow map,

$$
\Phi\left(\rho_{i}\right)=\left\{\begin{array}{rl}
v^{\text {free }} \rho_{i} & 0 \leq \rho_{i} \leq \rho^{\text {crit }} \\
w\left(\rho^{\max }-\rho_{i}\right) & \rho^{\text {crit }}<\rho_{i} \leq \rho^{\max }
\end{array},\right.
$$

and, its corresponding speed-density map,

$$
\Psi\left(\rho_{i}\right)=\left\{\begin{array}{cl}
v^{\text {free }} & 0 \leq \rho_{i} \leq \rho^{\text {crit }} \\
w\left(\frac{\rho^{\max }}{\rho_{i}}-1\right) & \rho^{\text {crit }}<\rho_{i} \leq \rho^{\max } .
\end{array}\right.
$$

Note that the admissible solutions are those satisfying the Riemann problem [9]. The Riemann's admissible solutions can be rewritten using the Demand-Supply formalism, where the demands $D\left(\rho_{i}\right)$ and supplies $S\left(\rho_{i}\right)$ functions are given as (see also Fig 1):

$$
\begin{aligned}
& D\left(\rho_{i}\right)=\min \left(v^{\text {free }} \rho_{i}, \varphi^{\max }\right), \\
& S\left(\rho_{i}\right)=\min \left(\varphi^{\max }, w\left(\rho^{\max }-\rho_{i}\right)\right) .
\end{aligned}
$$

In a simple junction of one inflow and one outflow, the interface flow corresponds to

$$
\varphi_{i}^{\text {out }}=\min \left(D\left(\rho_{i}\right), S\left(\rho_{i+1}\right)\right),
$$

and the solutions $\varphi_{i}^{\text {out }}$ can be expressed as upper bounds of the inequalities

$$
\begin{aligned}
& 0 \leq \varphi_{i}^{\text {out }} \leq D\left(\rho_{i}\right) \\
& 0 \leq \varphi_{i}^{\text {out }} \leq S\left(\rho_{i+1}\right) .
\end{aligned}
$$

Maximizing the throughput as suggested by rule B), implies maximizing

$$
\max _{\varphi^{\text {out }}} \sum_{i=1}^{n} \varphi_{i}^{\text {out }}
$$

and introducing rule $\mathrm{A}$ ), implies that inflows and outflows are linearly related by the relation $\varphi_{j}^{\text {in }}=\sum_{i \in \mathcal{I}^{-}} r_{i j} \varphi_{i}^{\text {out }}$, transforming the inflows in

$$
\varphi^{\text {in }}=R^{T} \varphi^{\text {out }}+\varphi^{\text {ext }} .
$$

where $\varphi^{\text {ext }}=B \lambda_{e}$ is a vector containing exogenous external inflows to the network in the corresponding positions of $\varphi^{\text {in }}$ and zeros elsewhere. $B$ is a selection matrix for the incoming boundary flows of the network.

In problem (10) the flow $\varphi^{\text {out }}$ should respect the relationships (9) for each one of the entering roads to the intersection. Let us consider first an organization of the set of constraints for a single junction $g$ as

$$
\begin{gathered}
\check{\mathcal{D}}_{g}:\left\{\varphi_{i}^{\text {out }} \leq v^{\text {free }} \rho_{i} \quad \forall \quad i \in \mathcal{I}^{-}\right\}, \\
\mathcal{D}_{g}^{\max }:\left\{\varphi_{i}^{\text {out }} \leq \varphi^{\max } \quad \forall \quad i \in \mathcal{I}^{-}\right\}, \\
\check{\mathcal{S}}_{g}:\left\{\sum_{i=1}^{n} r_{i j} \varphi_{j}^{\text {out }} \leq w\left(\rho^{\max }-\rho_{j}\right) \quad \forall \quad j \in \mathcal{I}^{+}\right\}, \\
\mathcal{S}_{g}^{\max }:\left\{\sum_{j=1}^{n} r_{i j} \varphi_{j}^{\text {out }} \leq \varphi^{\max } \quad \forall \quad j \in \mathcal{I}^{+}\right\} .
\end{gathered}
$$

The set of constraints $\mathcal{D}_{g}=\check{\mathcal{D}}_{g} \cap \mathcal{D}_{g}^{\max }$ and $\mathcal{S}_{g}=\check{\mathcal{S}}_{g} \cap \mathcal{S}_{g}^{\max }$, complete the formulation required for the junction problem. With $\mathcal{P}_{g}^{\varphi}: \mathcal{D}_{g} \cap \mathcal{S}_{g}$ the solution to the junction problem can then be finally stated as:

$$
\begin{array}{ll}
\max _{\varphi^{\text {out }}} & \sum_{i=1}^{n} \varphi_{i}^{\text {out }} \\
\text { s.t. } & \varphi^{\text {out }} \in \mathcal{P}_{g}^{\varphi} .
\end{array}
$$

Solutions of the optimization problem (14) typically reach the upper boundaries of the constraints (See Fig. 1), and they provide a single unique solution for cases where $m>n$.

\section{Full traffic network model}

The full network of roads can be described by a weighted directed graph $\mathcal{H}:=(\mathcal{I}, \mathcal{R})$. The graph is represented by the union of all tuples $\left(\mathcal{I}:=\cup_{g \in \mathcal{G}} \mathcal{I}_{g}, \mathcal{R}:=\cup_{g \in \mathcal{G}} \mathcal{R}_{g}\right)$ for each intersection resulting in $n_{H}$ roads $^{3}$.

The complete traffic network model combines, dynamic equations of the density evolution of each road (2), in its vector form

$$
\rho(k+1)=\rho(k)+T L^{-1}\left(\varphi^{\text {in }}(k)-\varphi^{\text {out }}(k)\right),
$$

or equivalently, using rule A),

$$
\rho(k+1)=\rho(k)+T L^{-1}\left(\left(R^{T}-I\right) \varphi^{\mathrm{out}}(k)+\varphi^{\mathrm{ext}}(k)\right),
$$

where $T$ the sampling time and $L=\operatorname{diag}\left\{l_{i}\right\}$, with the junction models compactly represented by a graph resulting form the union of multiple single junction problems.

Definition 2 (Network Junction Problem): The extension of the single junction problem to the network case is represented as the union of all local problems respecting simultaneously all the constraints imposed by the traffic model at each junction.

$$
\max _{\varphi^{\text {out }}} \mathbb{1}^{T} \varphi^{\text {out }} \quad \text { s.t. } \varphi^{\text {out }} \in \mathcal{P}^{\varphi} .
$$

The network junction problem is the solution to the optimization problem (17). The union of all problems is obtained through the maximization of the total throughput $\sum_{i=1}^{n_{H}} \varphi_{i}^{\text {out }}=$ $\mathbb{1}^{T} \varphi^{\text {out }}$ and the constraints $\mathcal{P}^{\varphi}:=\bigcup_{g \in \mathcal{G}} \mathcal{P}_{g}^{\varphi}$.

\section{DENSITY AND FLOW RECONSTRUCTION}

In this section we present the joint density and flow reconstruction problem. We first indicate the main data characteristics and their associated observation model.

\section{A. Observations model and its mathematical properties}

Let $\mathcal{I}_{F C D}$ be the set of roads where FCD are collected. FCD measurements can be considered to be available everywhere in the network, i.e. $\left|\mathcal{I}_{F C D}\right|=n_{H}$. They describe the average velocity at each road. On the other hand, let $I_{M L D}$ be the set where MLD data is collected. They measure outflows at the road ends where sensors are installed. Loop

\footnotetext{
${ }^{3}$ We consider, all the roads in the graph $\mathcal{H}$ to be re-labelled with a single index $i$ so that each road keeps a unique identifier.
} 
detectors are not available at all roads $\left|I_{M L D}\right|=N_{M}<n_{H}$. These measurements have the following observation models,

$$
\begin{aligned}
\bar{v}_{i}(k) & =\Psi\left(\rho_{i}, k\right)+\eta_{v}\left(\rho_{i}, k\right), \quad i \in \mathcal{I}_{F C D} \\
\bar{\varphi}_{l}^{\text {out }}(k) & =\Phi\left(\rho_{l}, k\right)+\eta_{\varphi^{\text {out }}}\left(\rho_{l}, k\right), \quad l \in \mathcal{I}_{M L D}
\end{aligned}
$$

where the terms $\eta_{\varphi^{\text {out }}}, \eta_{v}$ represent additive noise produced by factors such as aggregation time, penetration rate, measurement noise, etc, which naturally may affect the measurement quality. The velocity measurement (18) will be used as a basis for density reconstruction as velocity are sensed in all the network road. However, its inverse map (noise apart)

$$
\rho_{i}=\Psi^{-1}\left(\bar{v}_{i}+\eta_{v}\right)
$$

is not invertible in the free-flow part (See Fig. 1). From other hand the inverse map of the flow,

$$
\rho_{l}=\Phi^{-1}\left(\bar{\varphi}_{l}^{\text {out }}+\eta_{\varphi^{\text {out }}}\right)
$$

has the problem that is not uniquely defined and that flow measures are spatially sparse. The non-unicity of (21) can be tackled by using the velocity measures allowing to discriminate congested from free-flow regimes, but can only be used in some roads. Therefore, velocity measurements and flow measurements will be used in different way to facilitate the signal reconstructions. The density/flow reconstruction algorithm is designed in the basis of the following assumptions:

Assumption 1 (Boundary flows): All inflows and outflows at the network boundaries are measurable.

Assumption 2 (Measured FCD speeds): Speeds captured by FCD are measured everywhere in the network.

Assumption 3 (Density pseudo-observation): Consider the fundamental diagram. Consider a measurement of speed measured in the congested regime, then there exists a density observation which can be uniquely recovered from the map (5), $\forall \rho_{i} \in\left[\rho^{\text {crit }}, \rho^{\max }\right], \forall \bar{v}_{i} \in\left[0, v^{\text {free }}\right)$

$$
\bar{\rho}_{i}=\Psi^{-1}\left(\bar{v}_{i}\right)=\frac{\rho^{\max }}{1+\bar{v}_{i} / w},
$$

Assumption 4 (Network Equilibrium): The reconstruction is done in a fast enough time scale so that the network can be considered to be at the equilibrium. At the network equilibrium, the flows are then related by the steady-state equation of (16),

$$
\left(R^{T}-I\right) \varphi^{\text {out }}+B \lambda_{e}=0 .
$$

Remark: Under full rank conditions for the matrix $R^{T}-I$ a unique solution $\varphi^{\text {out }}=-\left(R^{T}-I\right)^{-1} B \lambda_{e}$ can be obtained for the vector of outflows in the traffic network.

\section{RECONSTRUCTION PROBLEM}

We consider the reconstruction problem expressed as the solution to an optimization problem at a fixed time instant

$$
\min _{\hat{\varphi}^{\text {out }, \hat{\rho}}} \quad J_{\varphi^{\text {out }}}+J_{\rho} \quad \text { s.t } \mathcal{M}_{\varphi, \rho}
$$

where $\mathcal{M}_{\varphi, \rho}$ denotes a RTN model. The terms $J_{\varphi^{\text {out }}}$ and $J_{\rho}$ represent penalty functions for the error between the estimated values $\hat{\varphi}^{\text {out }}, \hat{\rho}$ and the measurements from the observation model in the systems.
The integration of these penalty functions allow the system to consider the reality captured by the observation model (20),(21). In particular, $J_{\varphi}$ integrates into the problem direct information contained in the MLD measurements. This cost function minimizes the error between available measurements $\varphi^{\overline{\text { out }}}=\left\{\bar{\varphi}_{l}^{\text {out }}, l \in \mathcal{I}_{M L D}\right\}$ through the quadratic norm $\sum_{l \in \mathcal{I}_{M L D}}\left(\hat{\varphi}_{l}^{\text {out }}-\bar{\varphi}_{l}^{\text {out }}\right)^{2}$.

On the other hand, assumption (3) allows a good recovery of information in the congested zones. According to Fig. 1 density values on congested zones are contained in the speed measurements. Let us consider

$$
S_{\bar{v}_{i}}=\left\{\begin{array}{l}
1, \quad \bar{v}_{i}<v^{\text {free }} \\
0, \text { elsewhere }
\end{array}\right.
$$

For all the network the selection matrix $S_{\bar{v}}=\operatorname{diag}\left\{S_{\bar{v}_{i}}\right\}$ is a transformation that contains on its diagonal ones for cells in congested zones and zeros everywhere else. The penalty goal for the density case is achieved by considering $\sum_{\bar{v}_{j}<\text { free }}\left(\hat{\rho}_{j}-\bar{\rho}_{j}\right)^{2}$, which has as an objective the minimization of density error in congested measured links. Finally the two terms can be exchanged transforming the problem into

$$
\min _{\hat{\varphi}^{\text {out }, \hat{\rho}}} \sum_{i=1}^{N_{M}}\left(\hat{\varphi}_{l}^{\text {out }}-\bar{\varphi}_{l}^{\text {out }}\right)^{2}+\sum_{j=1}^{N_{H}}\left(S_{\bar{v}_{j}}\left(\hat{\rho}_{j}-\bar{\rho}_{j}\right)\right)^{2} \text { s.t } \mathcal{M}_{\hat{\varphi}, \rho}
$$

a) Difficulties and relaxation of the estimation problem: Some difficulties lie in the formulation of the problem (25). Non linearities of $\mathcal{M}_{\varphi, \rho}$ may appear in the fundamental diagram and no explicit solution is known to the problem. When introducing non linear constraints to the estimation problem, the solutions are regularly hard to approximate and most algorithms extract local minima. In addition, the computational cost and the complexity to solve the problem increases when the size of the network augments.

We introduce then a relaxed version of this problem which considers two main aspects. First, given the sparse nature of the flow measurements, and considering assumption 4 the steady state of the system constitutes an additional source of information to the flow reconstruction problem. This can be can be integrated into the cost function through the norm $\left\|\left(I-R^{T}\right) \varphi^{\text {out }}+B \lambda_{e}\right\|^{2}$.

Even though this term adds information to (25), it does not tackle the nonlinearity. In order to relax the non linear constraints we make use of piecewise linear fundamental diagrams (4), (5). The set of constraints is then transformed into linear equalities. They shape boundaries of the space of solutions established by the network junction problem. This aims to push solutions of the estimation within the fundamental diagram. Given assumption 2 the information about speeds provides a way to classify congested from freeflow cell constraints as:

$$
\begin{aligned}
& \overline{\mathcal{D}}_{g}:\left\{\varphi_{i}^{\text {out }}=v^{\text {free }} \rho_{i} \quad \forall \quad i \in \mathcal{I}^{-} \wedge \bar{v}_{i}=v^{\text {free }}\right\}, \\
& \overline{\mathcal{S}}_{g}:\left\{\sum_{i=1}^{n} r_{i j} \varphi_{j}^{\text {out }}=w\left(\rho^{\text {max }}-\rho_{j}\right) \quad \forall \quad j \in \mathcal{I}^{+} \wedge \bar{v}_{j}<v^{\text {free }}\right\},
\end{aligned}
$$




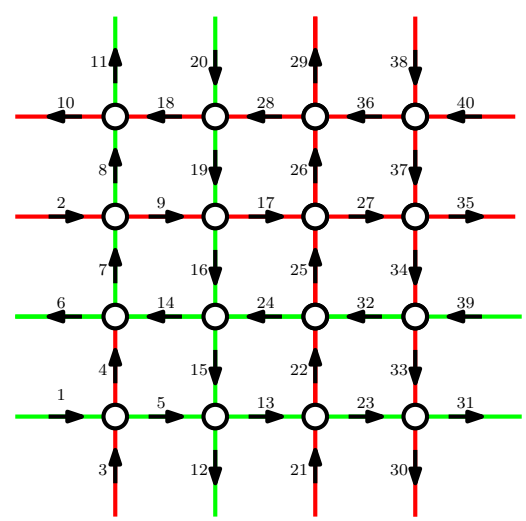

Fig. 3: Manhattan network topology under study

Such constraints can be expressed in terms of the selection matrix $S_{\bar{v}}$

$$
A(\bar{v}) \varphi^{\text {out }}=B(\bar{v}) \rho+C(\bar{v}),
$$

where

$$
\begin{gathered}
A(\bar{v})=\left[\begin{array}{c}
S_{\bar{v}}-I \\
R^{T} S_{\bar{v}}
\end{array}\right], \quad B(\bar{v})=\left[\begin{array}{c}
v^{\mathrm{free}}\left(S_{\overline{\bar{v}}}-I\right) \\
-w S_{\bar{v}}
\end{array}\right], \\
C(\bar{v})=\left[\begin{array}{c}
0 \\
w \rho^{\max } \mathbb{1} S_{\bar{v}}
\end{array}\right] .
\end{gathered}
$$

The resulting optimization problem is quadratic with linear equalities which provide good properties for the solution. The reconstruction problem can be formulated as follows.

Problem 1 (Joint density / flow reconstruction): Given a set of measurements of flow $\bar{\varphi}^{\text {out }} \in \mathcal{I}_{M L D}$ and a set of speed measurements $\bar{v} \in \mathcal{I}$ the inverse problem defined to recover $\hat{\rho}, \varphi^{\text {out }}$ is given by the solution of the following optimization problem:

$$
\begin{array}{ll}
\min _{\varphi^{\hat{\text { out }}, \hat{\rho}}} & \left\|C_{M} \hat{\varphi}^{\text {out }}-\bar{\varphi}^{\text {out }}\right\|_{\gamma_{\varphi}}^{2}+\left\|S_{\bar{v}}\left(\hat{\rho}-\Psi^{-1}(\bar{v})\right)\right\|_{\gamma_{\rho}}^{2}+ \\
& \left\|\left(R^{T}-I\right) \hat{\varphi}^{\text {out }}+B \lambda_{e}\right\|_{\gamma}^{2} \\
\text { s.t. } & A(\bar{v}) \hat{\varphi}^{\text {out }}=B(\bar{v}) \hat{\rho}+C(\bar{v}) \\
& \hat{\rho} \in \mathcal{P}^{\rho} .
\end{array}
$$

In (29) $\mathcal{P}^{\rho}:=\bigcup_{g \in \mathcal{G}} \mathcal{P}_{g}^{\rho}$ define the boundaries for $\rho$. In this case this particular boundaries can be determined from the speed measurements $\bar{v} . C_{M}$ selection matrix for the outgoing flows wherever they are available. The terms $\gamma_{\varphi}, \gamma_{\rho}, \gamma$ are weighting factors.

\section{SIMULATION SCENARIOS \& RESULTS}

\section{A. Scenario description}

We have built a manhattan topology network as depicted in Fig. 3. In this case, each one of the junctions is specified by 2 ingoing roads and 2 outgoing roads. The system is excited externally with maximum demands $\varphi^{\max }$ for external inflows and outflows selected from a uniform distribution in $\left[0, \varphi^{\max }\right]$, meaning the boundary conditions of the network are known. The selection of the splitting ratios is fixed so that $70 \%$ of the flow continues in a straight direction while the remaining turns. We consider all cells are uniform $l_{i}=500 \mathrm{~m}$, $v^{\text {free }}=50 \mathrm{Km} / \mathrm{h}, \varphi^{\max }=2000 \mathrm{veh} / \mathrm{h}$. The corresponding value

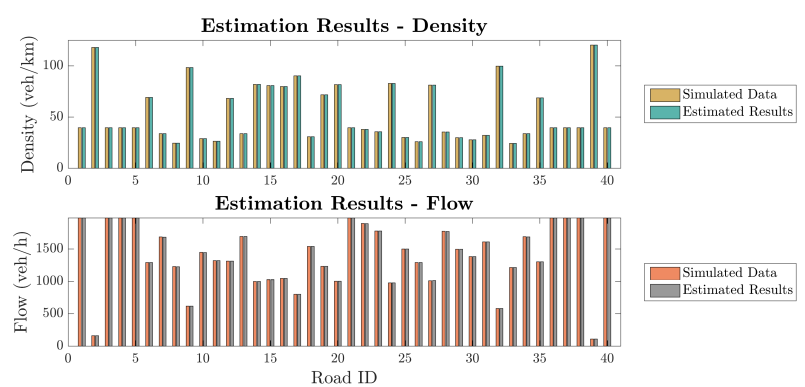

Fig. 4: Comparison of ground truth and estimated values for density (top) and flow (bottom) at network equilibrium

of $w$ is obtained from (4). For the purpose of this simulation $\gamma_{\varphi}=\gamma_{\rho}=\gamma=1$.

\section{B. Performance measurement}

In order to assess performance of the method, we consider the Absolute Error (AE) as a reference:

$$
\begin{gathered}
A P E_{\rho}(k)=\frac{1}{N} \sum_{i=1}^{N}\left|\rho_{i}(k)-\hat{\rho}_{i}(k)\right| \\
A P E_{\varphi^{\text {out }}}(k)=\frac{1}{N} \sum_{i=1}^{N}\left|\varphi_{i}^{\text {out }}(k)-\hat{\varphi}_{i}^{\text {out }}(k)\right|
\end{gathered}
$$

We illustrate the performance and limitations of the discussed estimation method. Initially, a simulation of the Manhattan grid using the CTM and the network junction problem solver was run until it reached equilibrium. These results were taken as ground truth. Using this equilibrium state, the density and flow reconstruction method was applied by using measurements of the inflows at the boundaries and speed information everywhere. Results are shown in Figure 4.

Even though the algorithm has been proposed for a static case, to illustrate the performance of the algorithm we consider a dynamical scenario. For this, a second experiment was carried out. For this instance, the traffic network was given an random initial condition of flow and density, and the inflows at the boundaries were initialized with a demand equal to random but fixed values in the interval $\left[0, \varphi^{\max }\right]$. Each of the iterations of the simulation process represents 15 seconds. At every step, the estimation method was applied with the assumption that the network had reached equilibrium. Results are presented in Fig. 5.As it can be observed, the estimation algorithm is able to capture the free-flow and congested locations of the network, with values close to ground truth. For the case of vehicle density, the mean absolute error is $0.025 \mathrm{veh} / \mathrm{km}$ with a maximum error of $0.037 \mathrm{veh} / \mathrm{km}$, whereas vehicle flow presented a mean error of $0.45 \mathrm{veh} / \mathrm{h}$ and a maximum error of $1.5 \mathrm{veh} / \mathrm{h}$. It should also be noted that for traffic flow the proposed method tends to approximates in a good way to the real values as desired.

\section{CONCLUSION}

This research has addressed the problem of joint density/flow reconstruction over urban traffic networks based 

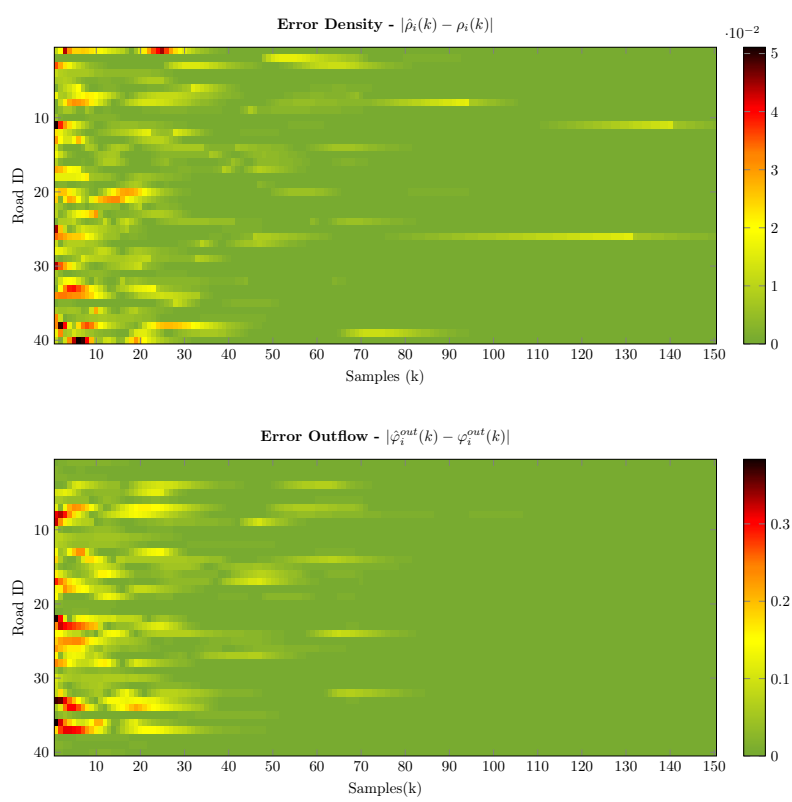

Fig. 5: Error dynamics for Flow and Density

on the fusion of multiple sources of information. The recovery process was achieved by introducing an estimation problem in which the cost function considers the integration of measurements and the network traffic model. We have introduced a relaxation to the original reconstruction problem and we have converted it into a quadratic problem under linear constraints which presents nice properties to be solved. The solutions of the problem show a good recovery in a static as well as in a dynamic case.

Future works on this aspect involves the validation of the current technique within a micro simulated scenario, the analysis of robustness of the method with respect to penetration rate, in particular the noise characterization related to the observability model of speeds. Other future works include the study the optimal sensor placement problem to improve the efficiency of the method and a study of the scalability of this model.

\section{ACKNOWLEDGMENT}

This work has been funded by the EU FP7 project SPEEDD (619435). This work has also received funding from the European Research Council (ERC) under the European Union's Horizon 2020 research and innovation programme (grant agreement $\mathrm{N}^{\circ} 694209$ ).

\section{REFERENCES}

[1] N. Bekiaris-Liberis, C. Roncoli, and M. Papageorgiou. Highway Traffic State Estimation With Mixed Connected and Conventional Vehicles. IEEE Transactions on Intelligent Transportation Systems, 17(12):3484-3497, dec 2016.

[2] S. Blandin, A. Couque, A. Bayen, and D. Work. On sequential data assimilation for scalar macroscopic traffic flow models. Physica D: Nonlinear Phenomena, 241(17):1421-1440, 2012.

[3] G. Bretti, R. Natalini, and B. Piccoli. Numerical approximations of a traffic flow model on networks. Networks and Heterogeneous Media, 1(1):57-84, 2006.
[4] E. S. Canepa and C. G. Claudel. Networked traffic state estimation involving mixed fixed-mobile sensor data using Hamilton-Jacobi equations, 2016.

[5] C. F. Daganzo. The cell transmission model: A dynamic representation of highway traffic consistent with the hydrodynamic theory. Transportation Research Part B, 28(4):269-287, 1994.

[6] C. F. Daganzo. The cell transmission model, part II: Network traffic. Transportation Research Part B, 29(2):79-93, apr 1995.

[7] W. Deng, H. Lei, and X. Zhou. Traffic state estimation and uncertainty quantification based on heterogeneous data sources: A three detector approach. Transportation Research Part B: Methodological, 57:132157, 2013.

[8] N.-E. E. Faouzi, H. Leung, and A. Kurian. Data fusion in intelligent transportation systems: Progress and challenges A survey. Information Fusion, 12(1):4-10, jan 2011.

[9] M. Garavello, K. Han, and B. Piccoli. Models for vehicular traffic on networks. American Institute of Mathematical Sciences, 2016.

[10] P. Grandinetti, F. Garin, and C. Canudas-De-Wit. Towards scalable optimal traffic control. In Proceedings of the IEEE Conference on Decision and Control, volume 2016-Febru, pages 2175-2180. IEEE, dec 2016.

[11] J.-P. Lebacque. The Godunov scheme and what it means for first order traffic flow models. International Symposium on Transportation and Traffic Theory, pages 647-677, 1996.

[12] M. J. Lighthill and G. B. Whitham. On Kinematic Waves. II. A Theory of Traffic Flow on Long Crowded Roads. Proceedings of the Royal Society A: Mathematical, Physical and Engineering Sciences, 229(1178):317-345, 1955.

[13] E. Lovisari, C. Canudas de Wit, and A. Y. Kibangou. Data fusion algorithms for density reconstruction in road transportation networks. In 2015 54th IEEE Conference on Decision and Control (CDC), pages 2804-2809, Osaka, dec 2015. IEEE.

[14] E. Lovisari, C. Canudas de Wit, and A. Y. Kibangou. Density/Flow reconstruction via heterogeneous sources and Optimal Sensor Placement in road networks. Transportation Research Part C: Emerging Technologies, 69:451-476, 2016.

[15] F. Morbidi, L. L. Ojeda, C. Canudas de Wit, and I. Bellicot. A new robust approach for highway traffic density estimation. In 2014 European Control Conference (ECC), pages 2575-2580, Strasbourg, France, jun 2014. IEEE.

[16] G. Newell. A simplified theory of kinematic waves in highway traffic, part II: Queueing at freeway bottlenecks. Transportation Research Part B: Methodological, 27(4):289-303, aug 1993.

[17] P. I. Richards. Shock Waves on the Highway. Operations Research, 4(1):42-51, 1956.

[18] T. Seo, A. M. Bayen, T. Kusakabe, and Y. Asakura. Traffic state estimation on highway: A comprehensive survey. Annual Reviews in Control, 43:128-151, 2017.

[19] Y. Wang, M. Papageorgiou, and A. Messmer. RENAISSANCE - A unified macroscopic model-based approach to real-time freeway network traffic surveillance. Transportation Research Part C: Emerging Technologies, 14(3):190-212, jun 2006.

[20] Y. Wang, M. Papageorgiou, A. Messmer, P. Coppola, A. Tzimitsi, and A. Nuzzolo. An adaptive freeway traffic state estimator. Automatica, 45(1):10-24, jan 2009.

[21] D. B. Work, S. Blandin, O. P. Tossavainen, B. Piccoli, and A. Bayen. A Traffic Model for Velocity Data Assimilation. Applied Mathematics Research eXpress, 2010(1):1-35, 2010. 